\title{
Histogram comparison crosscalibration method applied for evaluating the quality of "Canopus-v-ir" data
}

\author{
Mikhail Burcev ${ }^{1}$, Alexandr Kashnitskiy ${ }^{1}$, Evgeny Loupian ${ }^{1}$, Alexey Mazurov ${ }^{1}$, \\ Ilya Pustynskiy $^{2}$, and Egor Kholodov ${ }^{2}$ \\ ${ }^{1}$ Space Research Institute, Russian Academy of Sciences, Moscow, Russia \\ ${ }^{2}$ Far-Eastern center SRC "Planeta", Khabarovsk, Russia
}

\begin{abstract}
Regular onboard calibration of satellite data is of great practical importance for the processing of remote sensing information. For flight cross-calibration it is proposed to use a geostationary satellite as a reference to calibrate polar orbiting satellites data on the example of the "Canopus-V-IR" and Himawary satellites. Authors use data from center for collective use "IKI-Monitoring", united data catalogue of Planeta Research Centers for Space Hydrometeorology. The work has been done on theme «Monitoring»
\end{abstract}

Modern remote sensing devices are the measuring instruments that provide data used in in various tasks of global changes monitoring in the earth's surface and determining dangerous phenomena. The accuracy and stability of the calibration characteristics of satellite instruments are important for the development and sustainable automatic application of processing algorithms. During the operation of spacecraft, it is necessary to conduct regular flight calibration of instruments, for which various techniques are used [14].

The method proposed for use is based on the comparison of histograms of images from the same Earth's surface scene by instruments from different spacecraft having similar spectral characteristics and made with the same view geometry and at the same time [5]. One of the devices will be considered a reference, with good calibration. We will try to calibrate other devices according to that one.

It seems natural to assume that the sensitivity of the device has no inversion and that the magnitude of the signal recorded by the device is proportional to the value of the radiation flux received from the Earth, i.e. a brighter object on earth corresponds to a stronger signal recorded by the device. In this case, all objects with the same brightness should have the same area in the images from different devices, which in terms of the image means the same histogram value in the area corresponding to the brightness of the objects.

Thus, constructing the correspondence of the partial areas of the histograms, we obtain an unambiguous correspondence of the brightnesses of one image to the brightnesses of another, or in other words, the correspondence between the recorded values of one device and the registered values of another. With a good calibration of the reference device, we get a calibration table for the recorded values of another device. 
One of the difficulties of such a scheme is the condition for simultaneous shooting with the same geometry. Nevertheless, when analyzing images of orbital and geostationary spacecraft, one can find approximate fulfillment of both the conditions of the geometry of the view and the conditions of simultaneity with an accuracy of less than 5 minutes, and more accurately with planning the session.

The study was conducted using data access and processing services "VEGA-Science" [6]. For the Canopus-V-IR data, scenes near to Himawari subsatillite point were provided by "VNIIEM Corporation" JC for April 12-15, 2019. Himawari data processed at the Far Eastern center SRC "Planeta" [7] were used as a reference.

The geometry of the both views of the scene from the geostationary and polar-orbiting satellite is shown in Fig. 1. On the images taken from two satellite the areas are selected to satisfy the geometry with difference of up to 5 degrees of satellites zenith angles.

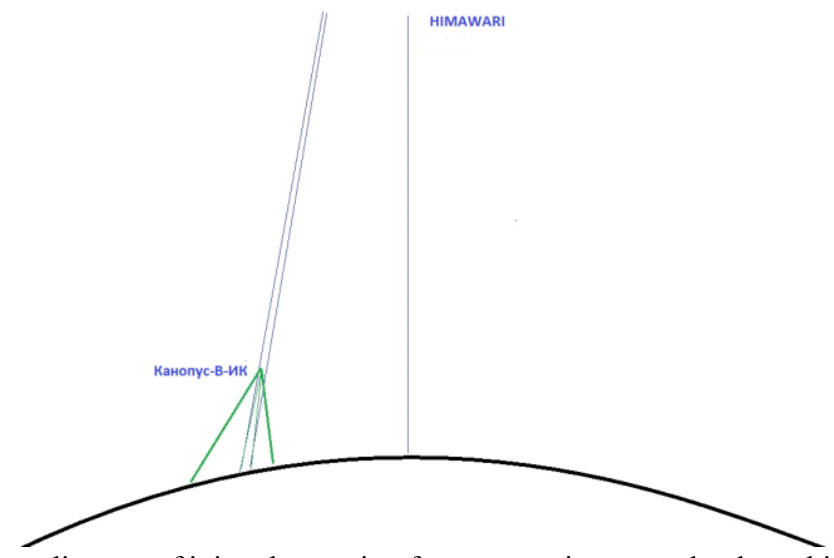

Fig 1. . Geometry diagram of joint observation from geostationary and polar-orbiting satellite.

Images by Canopus-V-IR are averaged to the images resolution of the AHI HIMAWARI satellite (these data are used as reference data). Further, according to the histograms of images using the method described above the calibration tables are calculated for the data "Canopus-_V-IK", MSU-IK-SRM device.

The spectral characteristics of the devices in the bands of $8.6 \mu \mathrm{m}$ and $3.7 \mu \mathrm{m}$ are shown in Fig. 2
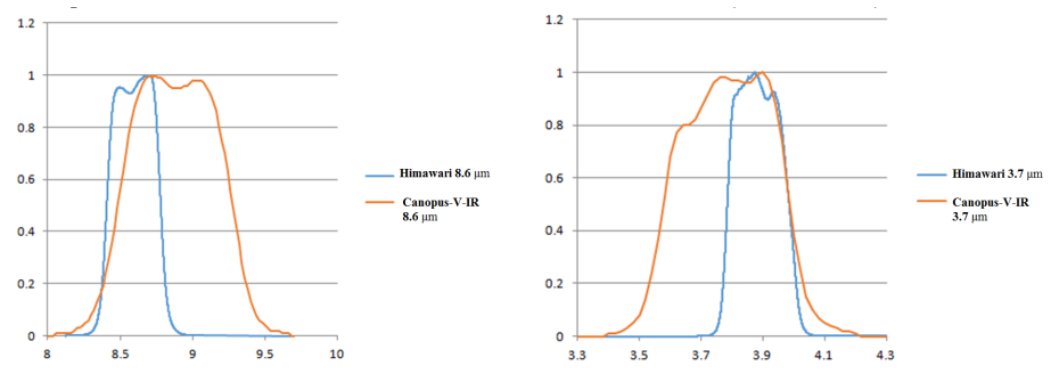

Fig 2 Spectral response sensitivities.

An example of a pair of images used for cross-calibration with a selected fragment for histogram analysis is shown in Fig. 3. The corresponding histograms and data conversion graph (calibration table) are shown in Fig. 4. 


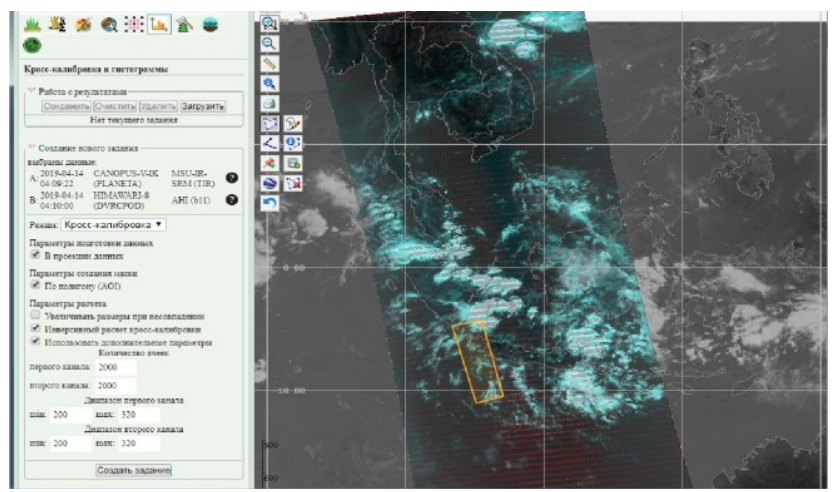

Fig 3. «Canopus-V-IR» and Himawari images mosaic with region for histogram analysis

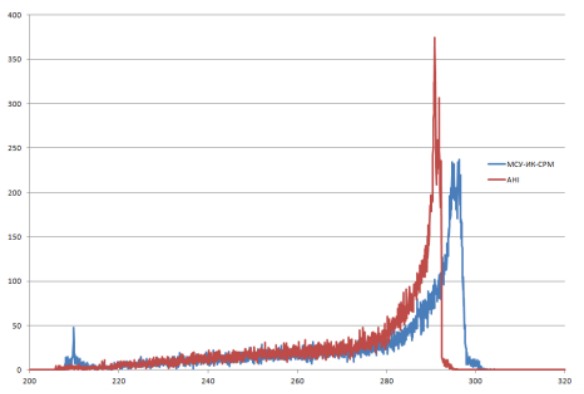

A.

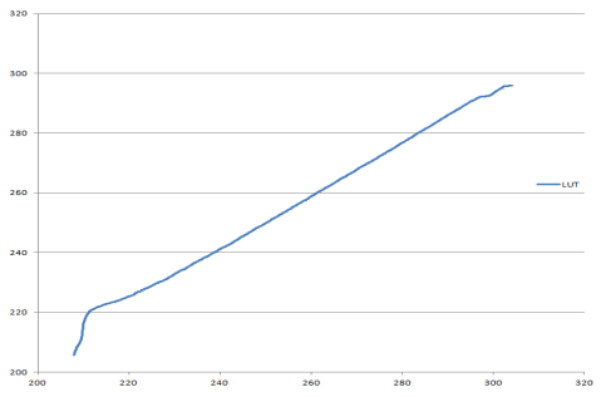

B.

Fig 4 Histogram.(A). Calibration table (B)

A number of factors affecting the proposed procedure should be noted. The Canopus-VIR data has obvious significant noise in the image (Fig. 5). This fact leads to errors in the construction of calibration tables. In particular, the apparent periodic strips in the warm zone (dark and irregular shades of the strip in the images Fig.56 a, b) overestimate part of the histogram of this region, this affects the conversion table in the upper right part of the graph of the calibration curve with higher than real temperature values and greater error in this area. Irregular values of other failures in the image (over the entire range of values) give corresponding errors to the conversion table, especially in places of the histogram with small values (in a colder area).

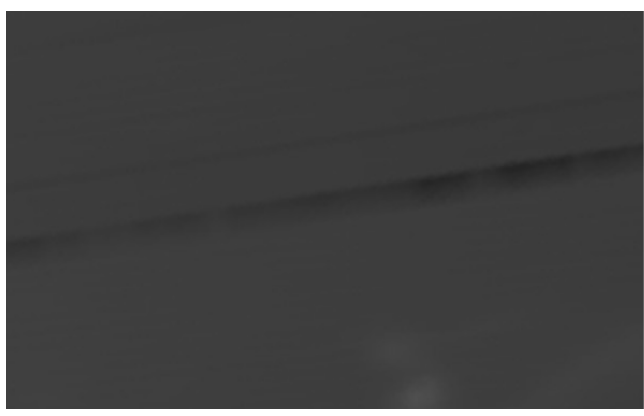

A

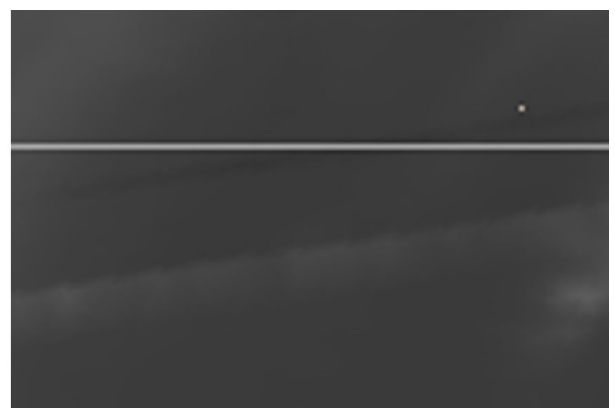

B

Fig 5. Noise strips over images. 
Calibration tables are constructed and averaged over a set of images (the graphs in abstract counts are shown in Fig. 6a and the result of the averaged table in terms of degrees $\mathrm{K}$ in Fig. 6b.
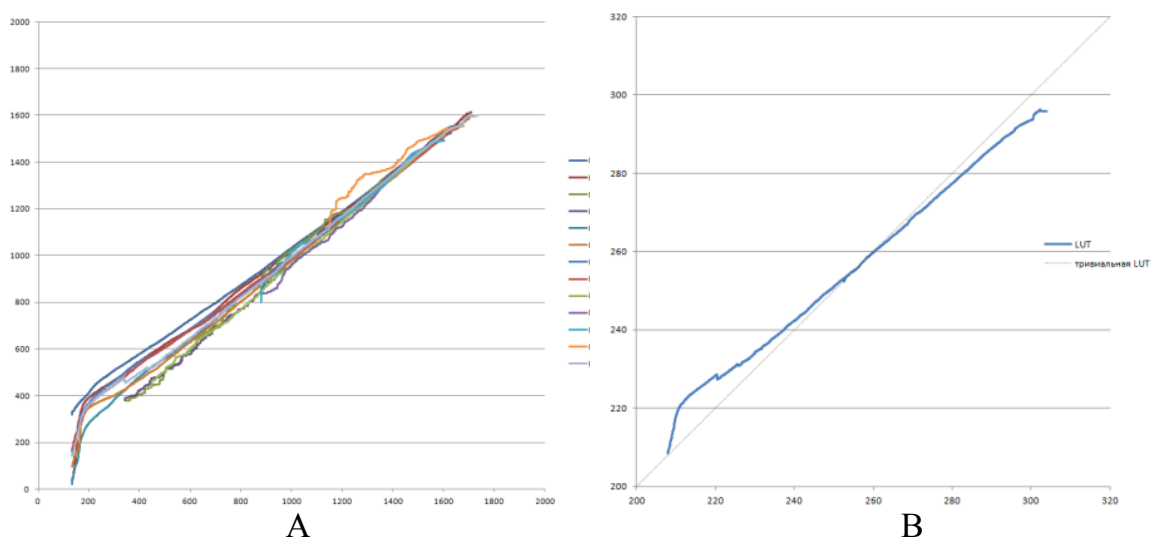

Fig 6.Calibration tables: set (A) and average (B).

The error (standard deviation) is from 1 (warm region) to 2.5 degrees $\mathrm{K}$ (cold region).

An almost linear course of the calibration curve should be noted, and only in the temperature range of $220 \mathrm{~K}$ and lower does the sensitivity drop, which appears in the histogram as a small upward surge in its values.

Sufficiently large deviations from the average are not associated with a possible sharp change in weather conditions in the considered regions within 5 minutes. For all data sets, the same conversion tables can be constructed for successive observations from the Himawari satellite. An example of the table graph for this case is shown in Fig. 7. It can be seen that it differs from the trivial linear transformation of the image itself, but not as much as the table graphs for the Canopus-V-IR-Himawari image pairs.

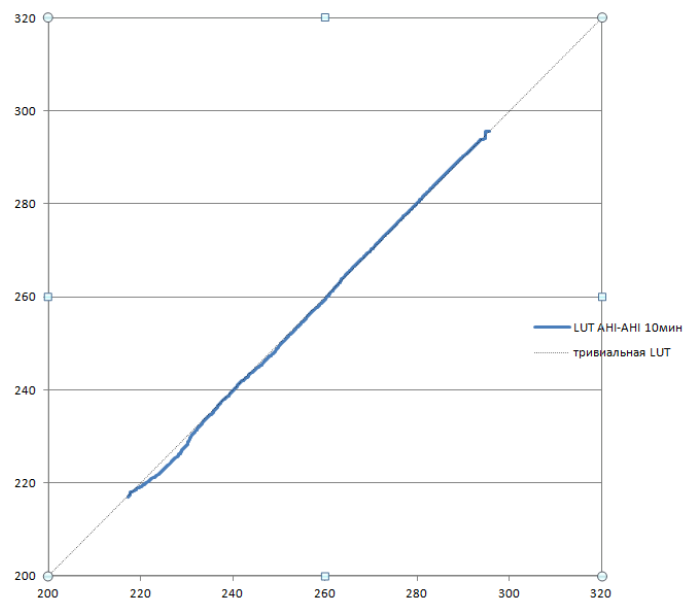

Fig 710 min delayed AHI images self-calibration table.

Thus, the error in the construction of refined calibration characteristics can be explained by the noise of the data. At the same time, the relatively close course of the calibration curves constructed using the above technique allows one to conclude that the Canopus-V- 
IR images after eliminating noisy data could be calibrated with using this cross-calibration technique.

The existing image set also contains data in a $3.7 \mu \mathrm{m}$ spectral band. But they are so noisy (Fig.8) that, due to the above considerations, this technique is not applicable for performing cross-calibration.

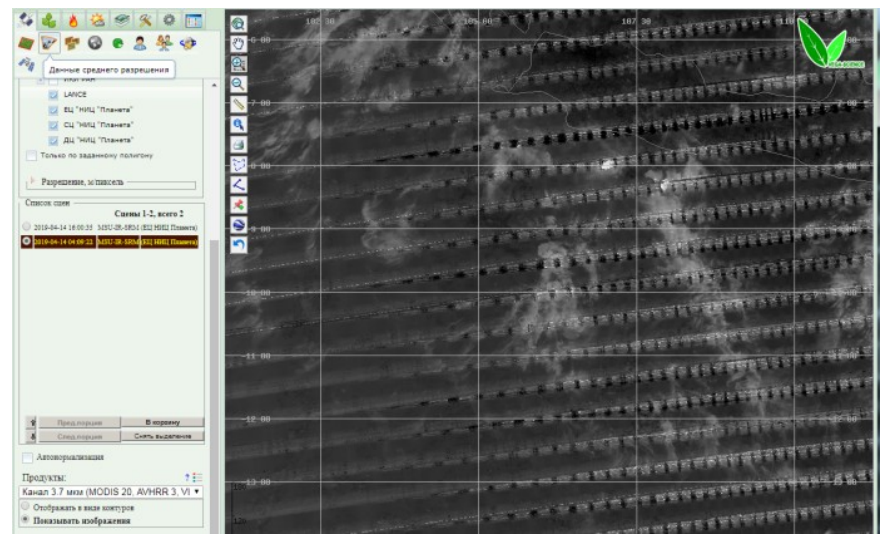

Fig 8 Canopus-V-IR image example at $3.7 \mu \mathrm{m}$.

Authors use data from center for collective use "IKI-Monitoring", united data catalogue of Planeta Research Centers for Space Hydrometeorology. The work has been done on theme «Monitoring»

1. G. Zhou, C. Li, T. Yue, L. Jiang, N. Liu, Y. Sun, M. Li An overview of in-orbit radiometric calibration oftypical satellite sensors, Remote Sensing and Spatial Information Sciences, 2015, Vol. XL-7/W4, 2015 International Workshop on Image and Data Fusion (2015)

2. G. Chander, T. J. Hewison, N. Fox, X. Wu, X. Xiong, J. William Overview of Intercalibration of Satellite Instruments, IEEE Transactions on geoscience and remote sensing (2013)

3. LiuJ.-J., Z. Li, Y.-L. Qiao, Y.-J Liu, Y.-X. Zhang A new method for cross-calibration of two satellite sensors, IEEE Int. J. Remote sensing (2004)

4. S. Sterckx, S. Adriaensen, W. Dierckx, M. Bouvet In-Orbit Radiometric Calibration and Stability Monitoring of the PROBA-V Instrument, Remote Sens. (2016)

5. A.V. Kashnitskiy, E.A. Loupian, A.A. Mazurov, A.M. Matveev The Possibility of Automatic Intercalibration for Russian Satellites by Histogram Analysis // "Information Technologies in Remote Sensing of the Earth-RORSE 2018". IKI RAS (2019)

6. E.A. Loupian, A.A. Proshin, M.A. Bourtsev, I.V. Balashov, S.A. Bartalev, V. Yu. Efremov, A.V. Kashnitskiy, A.A. Mazurov, A.M. Matveev, O.A. Sudneva, I.G. Sychugov, V.A. Tolpin, I.A. Uvarov IKI center for collective use of satellite data archiving,processing and analysis systems aimed at solving the problems of environmental study and monitoring // Actual Problems of Remote Sensing of the Earth from Space (2015)

7. E.A. Loupian, O.E. Milexin, V.N. Antonov, L.S. Kramareva, M.A. Bourtsev, I.V. Balashov, V.A. Tolpin, V.I. Solovyev System of operation of joint information resources based on satellite data in the Planeta Research Centers for Space Hydrometeorology // Russian Meteorology and Hydrology ( 2014) 